ARTICLE

\title{
An ester bond underlies the mechanical strength of a pathogen surface protein
}

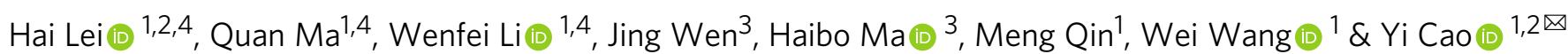

Gram-positive bacteria can resist large mechanical perturbations during their invasion and colonization by secreting various surface proteins with intramolecular isopeptide or ester bonds. Compared to isopeptide bonds, ester bonds are prone to hydrolysis. It remains elusive whether ester bonds can completely block mechanical extension similarly to isopeptide bonds, or whether ester bonds dissipate mechanical energy by bond rupture. Here, we show that an ester-bond containing stalk domain of $\mathrm{CpeO147}$ is inextensible even at forces $>2 \mathrm{nN}$. The ester bond locks the structure to a partially unfolded conformation, in which the ester bond remains largely water inaccessible. This allows the ester bond to withstand considerable mechanical forces and in turn prevent complete protein unfolding. However, the protecting effect might be reduced at non-physiological basic pHs or low calcium concentrations due to destabilizing the protein structures. Inspired by this design principle, we engineer a disulfide mutant resistant to mechanical unfolding under reducing conditions.

\footnotetext{
${ }^{1}$ Collaborative Innovation Center of Advanced Microstructures, National Laboratory of Solid State Microstructure, Department of Physics, Nanjing University, Nanjing, China. ${ }^{2}$ Chemistry and Biomedicine Innovation Center, Nanjing University, Nanjing, China. ${ }^{3}$ Key Laboratory of Mesoscopic Chemistry of MOE, School of Chemistry and Chemical Engineering, Institute of Theoretical and Computational Chemistry, Nanjing University, Nanjing, China. ${ }^{4}$ These authors contributed equally: Hai Lei, Quan Ma, Wenfei Li. ${ }^{凶}$ email: caoyi@nju.edu.cn
} 
G ram-positive bacteria produce a variety of surface adhesion proteins for host surface binding, biofilm formation, and immune evasion ${ }^{1-6}$. These proteins include rod-like pili and other large multidomain proteins such as microbial surface components recognizing adhesive matrix molecules $(\text { MSCRAMMs })^{7,8}$. Despite that these proteins have distinct synthesis pathways, they share similar 'beads on a string' extended organization. Many of the domains in these proteins adopt Ig-like structure with unusual intramolecular covalent crosslinks ${ }^{9,10}$, including thioester ${ }^{11-13}$, isopeptide ${ }^{14,15}$, and ester bonds ${ }^{16}$. These bonds endow the proteins outstanding mechanical functions for strong surface anchoring or resisting large mechanical perturbations. Depending on the chemical reactivity and the location in the protein structures, thioester and isopeptide bonds have distinct mechanical functions. Isopeptide bonds are generally formed between the side chains of Lys and Asn (or Asp) residues and are chemically inert under physiological conditions $s^{9,17-20}$. Both single-molecule force spectroscopy experiments $^{15,21,22}$ and molecular dynamics simulations ${ }^{23}$ revealed that isopeptide bond containing proteins can withstand considerable mechanical forces. For example, spy0128, the major pilin subunit of Streptococcus pyogenes, is inextensible up to $800 \mathrm{pN}$ due to the presence of isopeptide bond between the first and the last $\beta$ strands ${ }^{15}$. Moreover, as the isopeptide bond is located at the site of stress concentration, it locks the protein in a partially unfolded structure and allows fast refolding under $\operatorname{load}^{23}$. In another pilin subunit SpaA from Corynebacterium diphtheriae, the isopeptide bond can also restrict the unfolding of this CnaA Ig-type domain from complete unfolding under mechanical stress ${ }^{22}$. Unlike isopeptide bonds, thioester bonds are more labile and can react with amino groups that are abundant in host tissues ${ }^{12}$. They are often present at pilin tip-end adhesins and are formed in between the side chains of Cys and Gln residues. The reaction of thioester with nucleophilic ligands creates strong covalent adhesion with the host surfaces to resist large mechanical stresses ${ }^{12}$. Interestingly, recent studies revealed that the reactivity of thioester bonds is high at low forces and can be completely blocked at forces larger than $35 \mathrm{pN}$, entailing the bacteria stress dependent mobility ${ }^{24}$.

Ester bond is another type of intramolecular covalent bonds discovered in Gram-positive surface proteins and is formed between the side chains of Thr and Gln residues ${ }^{9,16,25}$. It was first discovered in a surface-anchoring protein Cpe0147 from Clostridium perfringens and then widely found in many bacterial surface adhesion molecules. The ester bonds in those proteins generally locate at the same position as the isopeptide bond in spy0128. However, as ester bonds are prone to hydrolyze at both acidic and basic conditions ${ }^{26}$, it is largely unknown how such unique chemical properties affect the mechanical properties of ester bond containing proteins. Previous studies showed that Cpe0147 is resistant to hydrolysis under normal physiological conditions, implying that the ester bond is shielded from the attack of $\mathrm{OH}^{-}$ions ${ }^{25}$. However, as mechanical force may locally destabilize the protein structures and then expose the buried ester bond to water, it remains elusive whether the ester bond containing proteins have distinct mechanical responses compared to the isopeptide bond containing proteins. Revealing the mechanical response of the ester bond containing proteins may also provide new insights into the pathological mechanism of Grampositive bacteria and help to develop antibiotics based on blocking the formation of ester bonds.

In this work, we employ atomic force microscopy (AFM)-based single-molecule force microscopy ${ }^{27-32}$, protein engineering, and molecular dynamics simulation to study the mechanical properties of the $\mathrm{C} 1$ domain of $\mathrm{Cpe} 0147$. We find that $\mathrm{C} 1$ exhibits similar mechanical stability as the isopeptide bond containing protein spy0128 and is inextensible even under forces $>2 \mathrm{nN}$. In contrast, if force is applied from a different orientation to unfold the protein structure, the ester bond breaks at forces of only $\sim 80 \mathrm{pN}$. These results suggest that the interplay between the ester bond and the protein structure is critical to the mechanical properties of the $\mathrm{C} 1$ domain. Molecular dynamics simulations reveal that the ester bond locks the structure to a partially unfolded conformation, in which the ester bond remains largely water inaccessible. This allows the ester bond to withstand considerable mechanical forces and in turn to prevent complete protein unfolding. Such a coupling between the ester bond and the protein structure is also found in another ester containing proteins, ParV of Parvimonas sp. Even when the ester bond is replaced by a disulfide, the mechanical stability of the protein is not affected. On the other hand, once the structure of the protein is destabilized at a basic $\mathrm{pH}$ or low calcium conditions, which are usually found on wound surfaces, some of the $\mathrm{C} 1$ domains can be unfolded, leading to the hydrolysis of the ester bond under mechanical load. Taken together, our studies reveal an uncharted mechanism to allow adhesion proteins to withstand high mechanical load using hydrolysable ester bonds. The environment-dependent mechanical stability may be related to the biological functions of Cpe0147 in vivo.

\section{Results}

Ester bond containing $\mathrm{Cl}$ domain is inextensible under mechanical forces $>\mathbf{2} \mathbf{~ n N}$. Cpe 0147 is a multiple-domain protein that covalently links the bacterial cell wall and the adhesin ${ }^{16}$ (Fig. 1a). The domains in the stalk region adopt similar all $\beta$ strand IgG-like fold and contains an ester bond linked the Thr on the first and the Gln on the last $\beta$-strands ${ }^{16}$ (Fig. 1b, c). To investigate the mechanical response of Cpe0147, we used the wide type $\mathrm{C} 1$ domain $\left(\mathrm{Cl}_{W T}\right)$ as the model system. We engineered a chimeric polyprotein, $\mathrm{Fg} \beta-(\mathrm{GB} 1)_{2}-\mathrm{Cl}_{W T}-(\mathrm{GB} 1)_{2}$-cys, for singlemolecule AFM experiments (Fig. 1d), following the experimental protocol reported by Yu et al. ${ }^{27}$. This polyprotein features specific noncovalent/covalent linkage of the polyprotein to either the cantilever tip or the substrate as well as mechanical fingerprint units for unambiguously identifying single-molecule events. The proteins were covalently linked to the substrate through the thiol group of the C-terminal Cys and then picked up by the SdrG modified cantilever tip through strong noncovalent interactions between $\mathrm{Fg} \beta$ and SdrG (rupture forces $>2 \mathrm{nN})^{28,33}$. Each end of $\mathrm{C1}_{W T}$ was flanked with two GB1 domains. The mechanical unfolding of GB1 was characterized by a contour length increment of $\sim 18 \mathrm{~nm}$ and an unfolding force of $\sim 200 \mathrm{pN}$ at a pulling speed of $1.6 \mu \mathrm{m} \mathrm{s}^{-134-36}$. Stretching the polyprotein allowed us to apply forces to $\mathrm{Cl}_{W T}$ between its $\mathrm{N}$ - and C-termini, similar to the force direction the protein domain experiences in their biological settings.

Stretching polyprotein led to representative sawtooth-like force-extension curves (Fig. 1f, Supplementary Fig. 1), in which each sawtooth peak corresponds to the force-induced unfolding of an individual domain in the polyprotein chain. However, we only observed five peaks; four of them showed the same contour length increments of $18 \mathrm{~nm}$, which can be attributed to the unfolding of the four GB1 domains. The last peak of more than $2 \mathrm{nN}$ arisen from the unbinding of the $\mathrm{Fg} \beta / \mathrm{SdrG}$ interaction ${ }^{28}$. There were no other peaks observed and the contour length of the last peak is about $130 \mathrm{~nm}$ (Supplementary Fig. 2) indicating that $\mathrm{C1}_{W T}$ did not fully unfold even under pulling forces higher than $2 \mathrm{nN}$. Note that, we observed a few events (24 in 805) with the first peak appeared at much longer contour length than normal ones, which may correspond to the proteins with the ester bond containing domain fully unfolded before stretching 

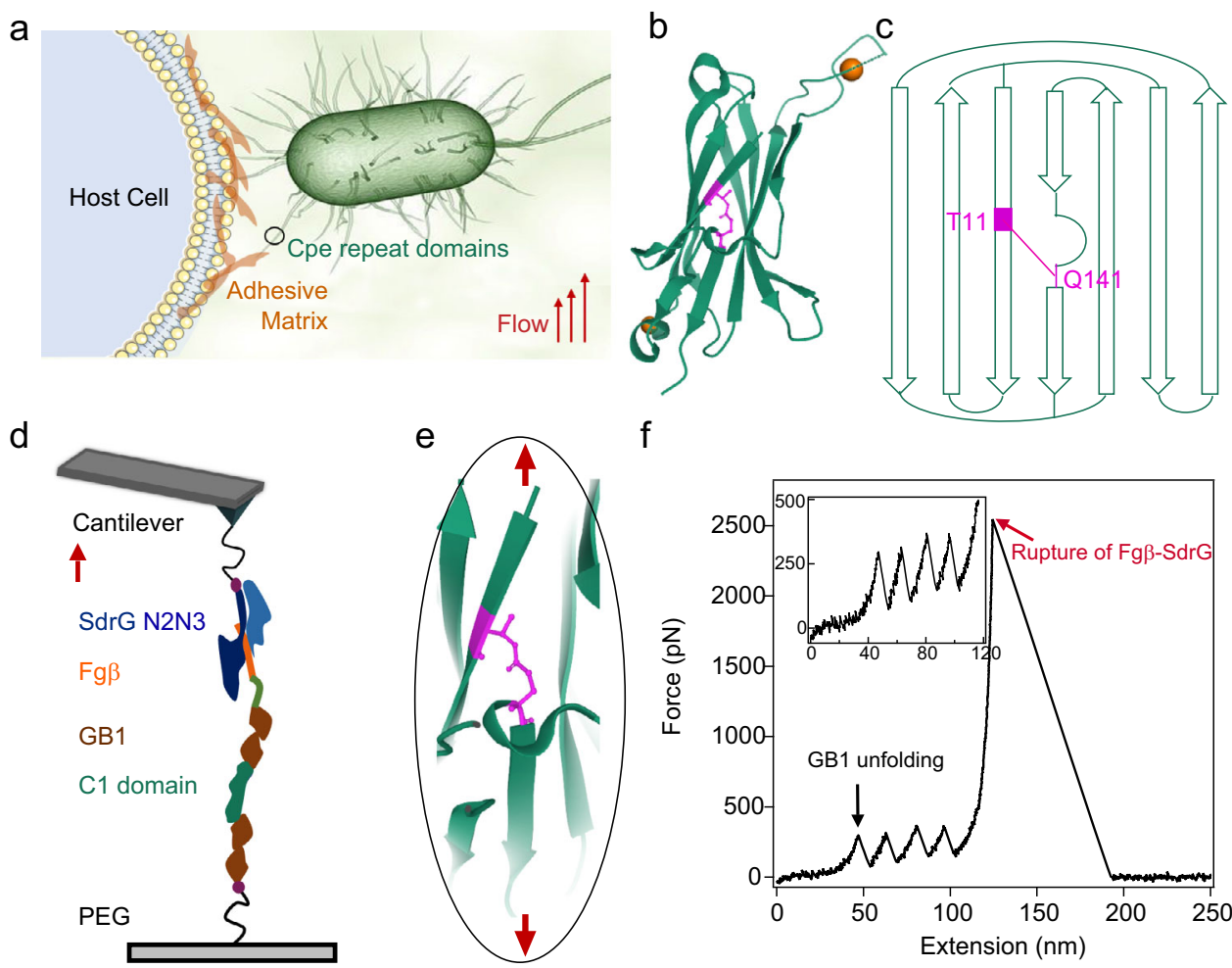

f

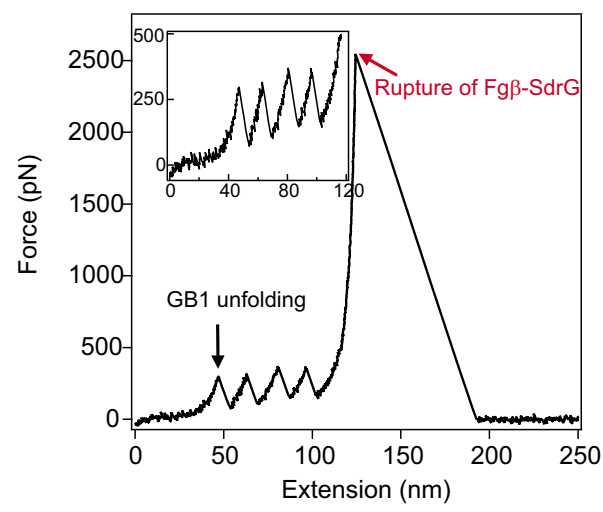

Fig. 1 Cpe0147 is highly resistant to mechanical forces. a Cpe0147 links the tip adhesion domain and bacteria to establish invasion and colonization and experiences considerable mechanical forces in the biological settings. b Structure of the C1 domain from PDB (4MKM). The ester bond is highlighted in magenta and the two calcium ions are shown as orange spheres. c Topology of $\mathrm{C1}_{W T}$ domain. The ester bond is formed between Thr- 11 and $\mathrm{Gln}-141$. $\mathbf{d}$ Schematic of the AFM-based single-molecule force spectroscopy experiments. Fg $\beta$-(GB1) ${ }_{2}-\mathrm{C1}_{W T}-(\mathrm{GB1})_{2}$-cys was covalently anchored to a glass surface through a polyethylene glycol (PEG) linker via thiol-maleimide chemistry and picked up using a SdrG-cys modified cantilever. e Mechanical extension of $\mathrm{C1}_{W T}$ from its $\mathrm{N}$ - and $\mathrm{C}$-termini is blocked by the ester bond as the ester bond is located right at the force concentration point. $\mathbf{f} \mathrm{A}$ representative singlemolecule force-extension trace at $1.6 \mu \mathrm{m} \mathrm{s}^{-1}$ showing the unfolding of the four GB1 domains (black arrow) at $200 \mathrm{pN}$ but no unfolding peak of $\mathrm{C} 1_{W T}$ up to the rupture forces of $\mathrm{Fg} \beta$-SdrG complexes (red arrow). This experiment was repeated more than six times independently with similar results. Source data are provided as a Source Data file.

(Supplementary Table 1). Increasing the pulling speed can increase the rupture forces of the $\mathrm{Fg} \beta / \mathrm{SdrG}$ complexes. However, we still did not observe the rupture of ester bonds (Supplementary Table 2 and Supplementary Fig. 3). We intended to further increase the maximum forces applied to $\mathrm{C} 1_{W T}$, by using covalent linkages to anchor the polyproteins to both the cantilever tip and the substrate. We constructed another polyprotein, Spytag-(GB1$\left.\mathrm{C1}_{W T}\right)_{4}$-cys. Spytag can bind Spycatcher to form a covalent isopeptide bond ${ }^{37,38}$. Unfortunately, the maximum detaching force in this design was even lower than that based on the Fg $\beta$ / SdrG interaction, presumably due to the maleimide-thiol chemistry we used for protein immobilization, which was not strong enough if the conjugate was not hydrolyzed ${ }^{39}$ (Supplementary Note 1). In this covalent linking scheme, each molecule can be stretched only once, preventing the mechanically triggered hydrolysis of maleimide-thiol adducts ${ }^{39}$ (Supplementary Note 2). Nonetheless, we only observed the unfolding events from four GB1 domains using this polyprotein (Supplementary Fig. 4). As the formation of intramolecular ester bond in the $\mathrm{Cl}$ domain was further confirmed by using the mass spectroscopy (Supplementary Fig. 5), it is safe to conclude that the wild-type C1 domain from Cpe0147 is inextensible up to the forces that can break weak covalent bonds.

Mechanical stability of the $\mathrm{C} 1$ domain without ester bond. To illustrate the role of ester bond in maintaining high mechanical stability of the $\mathrm{C} 1$ domain, we designed a protein variant $\mathrm{Cl}_{T 11 \mathrm{~A}}$ in which the ester bond was eliminated by mutating ester bond forming Thr at the 11th position to Ala (Fig. 2a, b) (Supplementary Fig. 6). Also, the polyprotein $\mathrm{Fg} \beta-(\mathrm{GB} 1)_{2}-\mathrm{Cl}_{\mathrm{Tl1}^{-}}$ $(\mathrm{GB} 1)_{2}$-cys was constructed and then studied using AFM-based single-molecule force spectroscopy. In the force-extension curves, besides the four peaks of $\Delta L_{c}$ of $\sim 18 \mathrm{~nm}$ for GB1, we observed an additional peak of $\Delta L_{c}$ of $47.4 \pm 3.25 \mathrm{~nm}$ (colored in red) (Fig. $2 \mathrm{c}$, Supplementary Fig. 7). The contour length increment is consistent with the complete mechanical unfolding of $\mathrm{C} 1\left(\Delta L_{c}=146\right.$ aa $\times 0.365 \mathrm{~nm} / \mathrm{aa}-5.2 \mathrm{~nm})$. The unfolding forces of $\mathrm{Cl}_{T 11 \mathrm{~A}}$ domain were about $92 \pm 41 \mathrm{pN}$ (Fig. 2e), which were lower than that of GB1. Eliminating the ester bond by the T11A mutation significantly reduced the unfolding forces of $\mathrm{C}_{W T}$. Therefore, the ester bond plays an important role in stabilizing the wild-type C1 structure under mechanical load.

Ester bond is mechanically weak in the absence of the folded C1 structure. To further understand the contribution of ester bond to the mechanical stability of $\mathrm{C} 1$, we design a circular permutant of $\mathrm{C} 1\left(\mathrm{Cl}_{C P}\right)$ to structurally decouple the rupture of ester bond and the unfolding of $\mathrm{Cl}_{W T}$. In $\mathrm{C} 1_{C P}$, the $\mathrm{N}$ - and C-termini of $\mathrm{C} 1$ were connected by an elastin-like peptide (ELP) of 45 amino acids, and the protein was split between the residues 125 and 126 to form the new N- and C-termini (Fig. 3a, b). Previously, Young et al. have shown that the two fragments obtained by splitting $\mathrm{C}_{W T}$ at this position can spontaneous rebind and form the inter-molecular ester bond ${ }^{25}$. Therefore, we anticipated that the ester bond can also formed in $\mathrm{Cl}_{C P}$. Mass spectroscopy analysis of $\mathrm{Cl}_{C P}$ confirmed the formation of the 

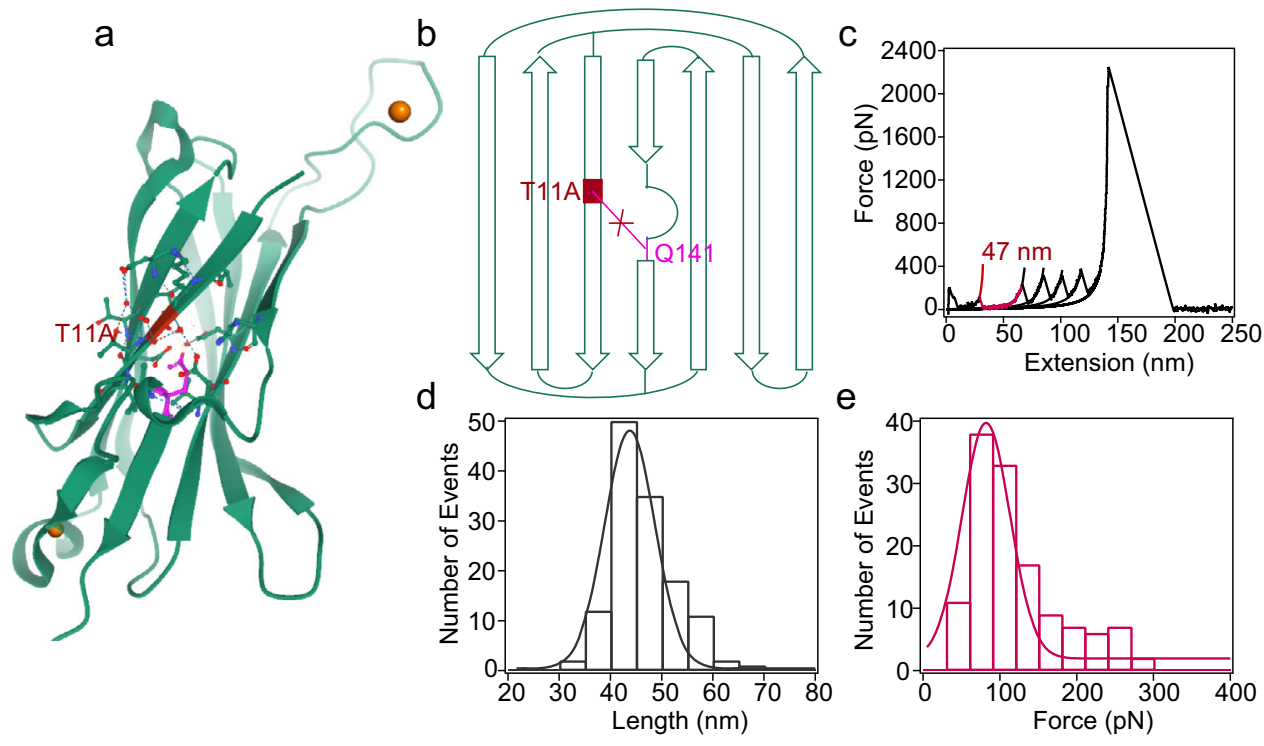

Fig. 2 Mechanical unfolding of the mutated C1 domain without the ester bond. a Thr-11 of $\mathrm{C} 1$ was mutated to Ala to eliminate the ester bond to yield the $\mathrm{C1}_{T 11 A}$ mutant. $\mathbf{b}$ Topology of $\mathrm{C1}_{T 11 A}$. The ester bond cannot form in the mutant. c Representative single-molecule force-extension curve of stretching Fg $\beta$ $(\mathrm{GB1})_{2}-\mathrm{Cl}_{\mathrm{T11A}^{-}}$-(GB1) ${ }_{2}$-cys following the same experimental protocol shown in Fig. 1d. Each peak was fitted by worm-like chain (WLC) model of polymer elasticity. The peak with a $\Delta L_{c}$ of $47 \mathrm{~nm}$ corresponds to the unfolding of $\mathrm{C1}_{T 11 \mathrm{~A}}$, the next four peaks correspond to the unfolding of GB1 domains, and the last peak corresponds to the rupture of the $\mathrm{Fg} \beta / \mathrm{SdrG}$ complex. $\mathbf{d}$ Histogram of contour length increment for $\mathrm{C} 1 \mathrm{unfolding}$ is centered $\sim 47 \mathrm{~nm}$. e Unfolding force histogram of $\mathrm{C} 1$ domain at a pulling speed of $1.6 \mu \mathrm{m} \mathrm{s}^{-1}$ measures an average unfolding force of $92 \pm 41 \mathrm{pN}(n=129$, total number of $\mathrm{C} 1$ unfolding events). Fitting the force distribution with Monte Carlo simulation ${ }^{65}$ results in a $\Delta \mathrm{x}_{\mathrm{u}}$ of $0.27 \mathrm{~nm}$ and $\alpha_{0}$ of $0.35 \mathrm{~s}^{-1}$ (Supplementary Fig. 8). This experiment was repeated three times independently with similar results. Source data are provided as a Source Data file.

ester bond (Supplementary Fig. 9). Note that in this new pulling direction, the ester bond is not located at the force concentration point (the position in the protein where the force is significantly higher than surrounding region) anymore. We would expect to see the rupture of ester bond after the unfolding of $\mathrm{Cl}_{C P}$, providing an unambiguously way to quantify the strength of the ester bond in the absence of the folded protein structure.

$\mathrm{Cl}_{C P}$ was stretched in the polyprotein $\mathrm{Fg} \beta-(\mathrm{GB} 1)_{2}-\mathrm{Cl}_{C P^{-}}$ $(\mathrm{GB} 1)_{2}$-cys construct and the representative force-extension curve is shown in Fig. $3 \mathrm{c}$ and Supplementary Fig. 10. In addition to the four peaks whose $\Delta L_{c}$ correspond to the unfolding of GB1, we observed two additional peaks of $\Delta L_{c}$ of $38 \pm 1.2 \mathrm{~nm}$ (colored in green) and $23 \pm 0.5 \mathrm{~nm}$ (colored in orange). The event in green always occurred first and then followed by the event in orange. Matching to the protein structure, these two events can be assigned to the piecewise extension of $\mathrm{Cl}_{C P}$ (Fig. 3d). The first peak corresponded to the unfolding of the protein and then extending the peptide sequence outside the ester bond (Thr-Gln). The measured $\Delta L_{c}$ matches well with the theoretically calculated value of $\sim 39 \mathrm{~nm}(((110-12)+(140-129))$ aa $\times 0.365 \mathrm{~nm} / \mathrm{aa}-0.9$ $\mathrm{nm}+0.6 \mathrm{~nm}$ ), where $0.9 \mathrm{~nm}$ is the distance between V113 and Q129 and $0.6 \mathrm{~nm}$ is the length of the ester bond containing linkage between T11 and Q141 (Fig. 3e). The unfolding forces of $C 1_{C P}$ were $91.6 \pm 52 \mathrm{pN}$ (Fig. 3f). The second peak resulted from the force-induced rupture of the ester bond and subsequently extending the peptide sequence shielded by this bond, as the measured $\Delta L_{c}$ of $23 \pm 0.5 \mathrm{~nm}$ matched well with the theoretically calculated value of $23 \mathrm{~nm}(66 \mathrm{aa} \times 0.365 \mathrm{~nm} / \mathrm{aa}-0.6 \mathrm{~nm})$ (Fig. $3 \mathrm{~g}$ ). Moreover, the $23-\mathrm{nm}$ peak was absent in the force-extension curves of the polyprotein containing $\mathrm{Cl}_{C P}$-T11A, in which $\mathrm{Thr}$ was mutated to Ala to eliminate the ester bond (Supplementary Figs. 11 and 12). This further demonstrated that the peaks of $\Delta L_{c}$ of $\sim 23 \mathrm{~nm}$ were indeed from the rupture of the ester bond in

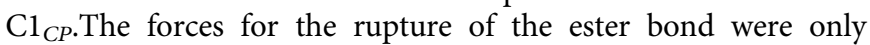
$77 \pm 56 \mathrm{pN}$ (Fig. 3h), much lower than that of typical covalent bonds ${ }^{40-43}$ and even some noncovalent bonds ${ }^{44-47}$. We inferred that this may be because the rupture of ester bond in water undergoes a hydrolysis mechanism ${ }^{26}$, distinct from the free radical homolysis mechanism for the rupture of other covalent bonds ${ }^{48}$. As indicated by the theoretical calculation by Akbulatov et al. ${ }^{26}$, ester hydrolysis under stretching follows a two-step kinetics. The first step involves the attack of the nucleophile $(\mathrm{OH}$ $-/ \mathrm{H}+$ ) to form a tetrahedral intermediate and the second step corresponds to the decomposition of the $\mathrm{C}-\mathrm{O}$ bond under force. The first step is typically the rate limiting step and shows a weak force-dependency with a free energy barrier within $1 \mathrm{kcal} \mathrm{mol}^{-1}$ based on the Bell-Evans model. The detailed ester hydrolysis mechanism under force may deserve further detailed experimental characterization, which is beyond the topic of this work. By repeatedly stretching and relaxing $\mathrm{Fg} \beta-(\mathrm{GB} 1)_{2}-\mathrm{Cl}_{\mathrm{CP}^{-}}(\mathrm{GB} 1)_{2}-$ cys, we were able to observe the reformation of the ester bond after rupture, suggesting that the ester bond rupture and formation are reversible under forces (Supplementary Fig. 13). These results clearly indicate that an unprotected ester bond is mechanically weak and the topology of the wild-type $\mathrm{C} 1$ structure is critical to its high mechanical stability.

Molecular dynamics simulations. To understand the molecular details underlying the ultrahigh mechanical stability of $\mathrm{C}$, we performed steered molecular dynamics simulations for both $\mathrm{C1}_{W T}$ and $\mathrm{Cl}_{C P}$ (Fig. 4a) (see "Methods" for more details). For $\mathrm{C1}_{W T}$ without force load, the ester bond was buried to a large extent and shielded from water attack by the nearby residues (Fig. 4b). When a constant pulling force of $1500 \mathrm{pN}$ was applied to the $\mathrm{N}$ - and C-terminal residues, the protein structure only partially unfolded at the force bearing first and last $\beta$-strands. However, as the ester bond was located at the force-concentrating point, it prevented force propagation to the rest parts of the protein and made the partially unfolded structure resemble the native structure (Fig. 4c). As the rupture of the ester bond requires water attack, the structural integrity of $\mathrm{C}_{W T}$ can in turn 
a

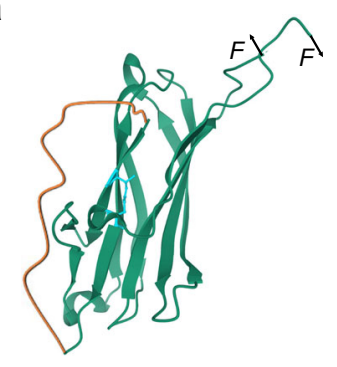

b

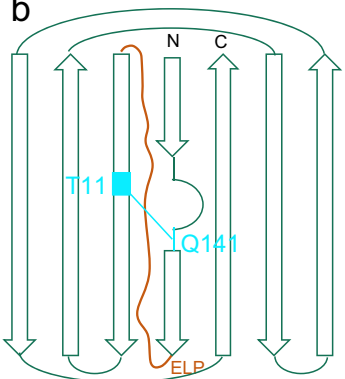

C

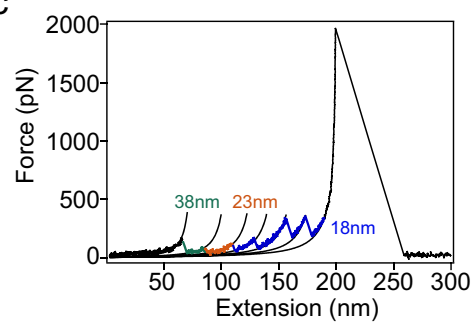

d

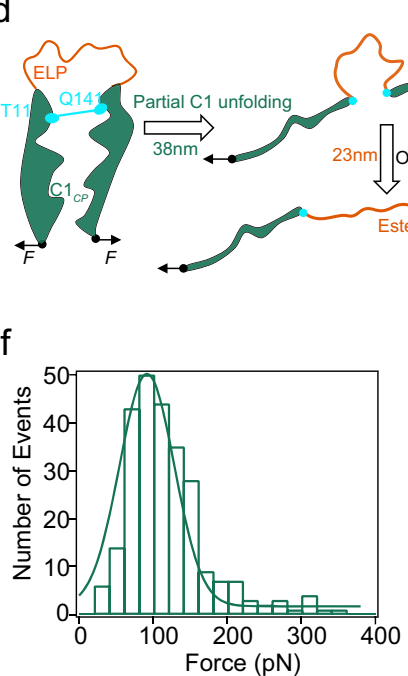

g

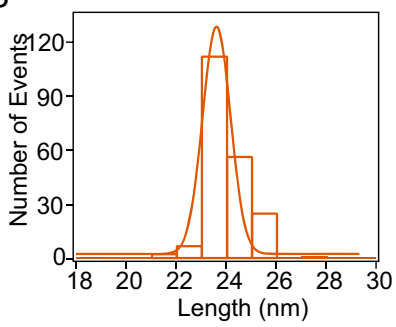

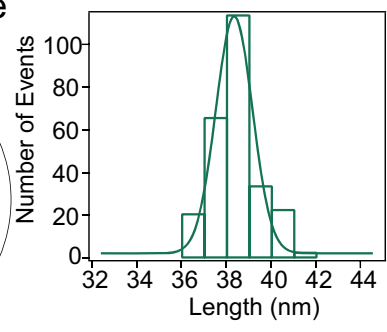

$\mathrm{h}$

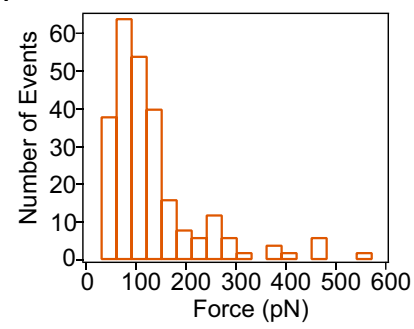

Fig. 3 Mechanical unfolding of the circular permutant of $\mathbf{C 1}, \mathbf{C 1}_{\mathbf{C P}}$. a Structure of $C 1_{C P}$ based on PDB (4MKM). $\mathbf{b}$ Topology of $C 1_{C P}$. Ester bond formed between T11 and Q141 is highlighted in cyan. The new N- and C-termini are at the position 126 and 125, respectively. The original $\mathrm{N}$ - and C-termini are connected by an ELP loop. c Representative force-extension curve of stretching $(\mathrm{GB} 1)_{2}-\mathrm{Cl}_{\mathrm{CP}}-(\mathrm{GB} 1)_{2}$ following the same experimental protocol shown in Fig. 1d. The force peaks with $\Delta L_{c}$ of $\sim 38 \mathrm{~nm}$ (green) and $\sim 23 \mathrm{~nm}$ (orange) are assigned as the unfolding of $C 1_{C P}$ and the rupture of ester bond, respectively. The force peaks of $\Delta L_{c}$ of $\sim 18 \mathrm{~nm}$ correspond to the unfolding of GB1 domains. $\mathbf{d}$ Schematic illustration of the contour length change upon stretching $C 1_{C P}$. First, the protein unfolds up to the ester bond position, then the rupture of ester bond releases the sequestered sequence. e Histogram of $\Delta L_{C}$ for $C 1_{C P}$ unfolding events peaks at $38 \mathrm{~nm}$. $\mathbf{f}$ Unfolding force histogram of $\mathrm{C1}_{C P}$ centers at $91.5 \pm 52 \mathrm{pN}(n=243$, total number of $\mathrm{C1}$ unfolding events). The Monte Carlo simulation of the force distribution results in a $\Delta \mathrm{x}_{\mathrm{u}}$ of $0.25 \mathrm{~nm}$ and $\alpha_{0}$ of $0.37 \mathrm{~s}^{-1}$ (Supplementary Fig. 14). $\mathrm{g}$ Histogram of $\Delta L_{c}$ corresponding to the rupture of ester bond in $\mathrm{Cl}_{C P}$ peaks at $23 \mathrm{~nm}$. $\mathbf{h}$ Histogram of the rupture forces of ester bond centers at $77 \pm 56 \mathrm{pN}$ ( $n=243$, total number of rupture of ester bond events). This experiment was repeated three times independently with similar results. Source data are provided as a Source Data file.

ensure high mechanical stability of the ester bond. In comparison, for $\mathrm{Cl}_{C P}$, the ester bond started to sustain the pulling force only at the later stage of the unfolding event. Applying pulling force led to complete protein unfolding and exposure of the ester bond to water (Fig. 4d). To estimate the reactivity of the ester bonds in the three conditions, we quantified the number of water molecules within $5 \AA$ from the Oe1 atom of the Gln-141. For $\mathrm{C} 1_{W T}$, even at a constant pulling force of $1.5 \mathrm{nN}$, the number of water molecules accessible to the ester bond was only slightly higher than that without force load (Fig. 4e). In contrast, for $\mathrm{Cl}_{C P}$, the ester bond was surrounded by $\sim 9$ water molecules due to the unfolding of the protein structure. Detailed structural analysis revealed that the bulky side chains of residues K10, T12, H133, D138, and A140 near the ester bond form a cage to shield the water molecules outside (Supplementary Fig. 15a). This cage structure was well maintained in $\mathrm{Cl}_{W T}$ even under a stretching force of $1.5 \mathrm{nN}$ and only slightly open at $2 \mathrm{nN}$, as indicated by the root-mean-square deviation (RMSD) of these residues (Supplementary Fig. 15b). However, in $\mathrm{C1}_{C P}$, this cage fell apart quickly, making the ester bond exposed to water molecules. Moreover, without the ester bond, even in $\mathrm{Cl}_{W T}$, the residues that form the ester bond were exposed to water molecules within a few hundreds of picoseconds (Supplementary Fig. 15c). The maximum number of water molecules was $\sim 14$, which was even higher than that for $\mathrm{Cl}_{C P}$ with the ester bond under forces. Comparing the distance evolution between the ester bond forming residues (Supplementary Fig. 15d) and the overall structural change of the protein in the absence of ester bond (Supplementary Figs. 15e and 16), we can clearly see that the separation of the ester bond forming residues was always prior to the increase of RMSD, indicating that breaking of the ester bond is a necessary step toward complete unfolding of the overall protein structure. Such results clearly demonstrated the mutual interplay between the chemical event and protein mechanical stability.

C1 structure as a "safe house" to protect mechanical rupture of disulfide bonds. Inspired by the molecular mechanism that allows the integration of labile ester bond and weak protein fold to achieve remarkable mechanical stability, we hypothesized that it is also possible to use the $\mathrm{C} 1$ structure as a mechanically robust cage or "safe house" to protect other chemically active bonds from attack. To this extend, we replaced the ester bond in $\mathrm{C} 1$ to disulfide bond 49,50 through T11C and Q141C mutation (the protein is named as C1-df hereafter), and studied the mechanical stability of this protein in the presence of reducing agent $(10 \mathrm{mM}$ tris (2-carboxyethyl) phosphine, TCEP, Supplementary Note 3). We built a polyprotein Fg $\beta$ - $(\mathrm{GB} 1)_{2}-\mathrm{C} 1-\mathrm{df}-(\mathrm{GB} 1)_{2}$-Spytag and 
a
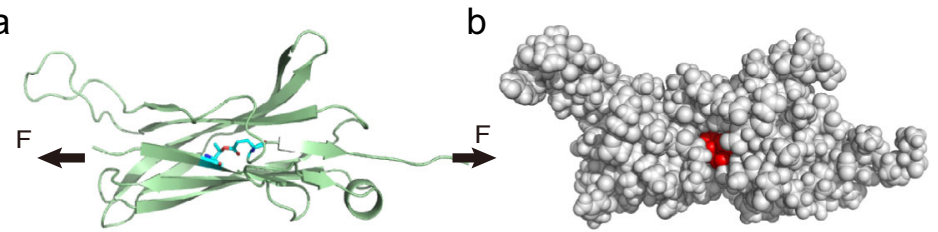

C
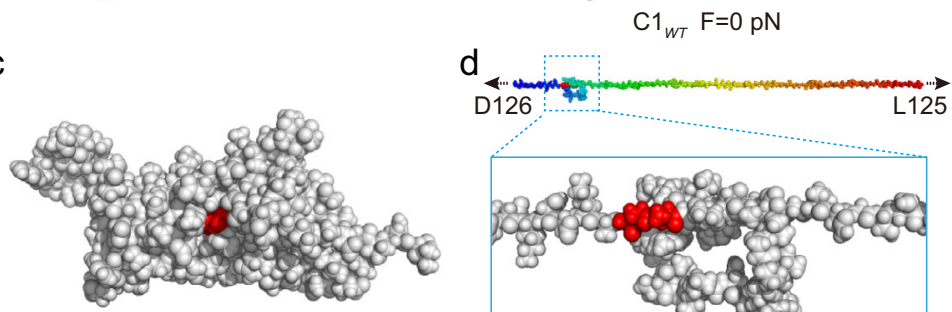

$\mathrm{C} 1_{W T} \mathrm{~F}=1500 \mathrm{pN}$

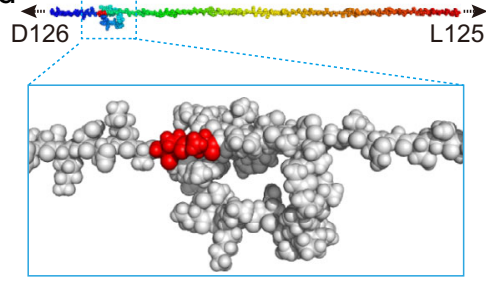

$\mathrm{C} 1_{C P} \mathrm{~F}=1500 \mathrm{pN}$

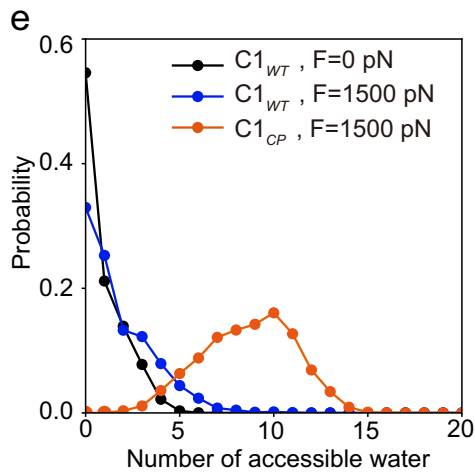

Fig. 4 Molecular dynamics simulations demonstrating different water accessibility of the ester bond in $\mathbf{C 1} 1_{w t}$ and $\mathbf{C 1}_{C P}$ under pulling force. a Cartoon representation of the three-dimensional structure of $\mathrm{Cl}_{W T}$ under the pulling force of $1500 \mathrm{pN}$. The residues Thr- 11 and $\mathrm{Gln}-141$ forming the ester bond were also shown by sticks representation. Three-dimensional structure of $\mathrm{C}_{W T}$ shown by sphere representation without force (b) and under the pulling force of $1500 \mathrm{pN}(\mathbf{c})$. d Three-dimensional structure of $\mathrm{Cl}_{C P}$ under the pulling force of $1500 \mathrm{pN}$ shown by sphere representation. For clarity, the zoom-in structure was also shown. The residues Thr-11 and Gln-141 were colored in red in b-d. e Distribution of the number of water molecules within $5 \AA$ from the Oع1 atom of the Gln-141 for $C 1_{W T}$ (blue) and $\mathrm{Cl}_{C P}$ (orange) at the constant pulling force of $1500 \mathrm{pN}$. For comparison, the result for $\mathrm{C} 1_{W T}$ (black) without applying force was also shown. Source data are provided as a Source Data file.

linked it to the cys-GB1-Spycatcher modified substrate by using Spycatcher/Spytag interaction instead of direct thiol-maleimide interaction to avoid the formation of mismatched disulfide bonds between the C-terminal Cys and Cys11/Cys141 that otherwise would complicate the single-molecule AFM experiments. The experimental scheme is shown in Fig. 5a. Note that the disulfide bond was not $100 \%$ formed in all proteins. Thus, the mechanical unfolding of $\mathrm{C} 1-\mathrm{df}$ could result in three different scenarios (Fig. 5b): (1) the unfolding of C1-df without the disulfide bond (denoted as the "Disulfide unformed" group); (2) the complete locking of $\mathrm{C} 1$-df with disulfide bond at the folded state without unfolding (denoted as the "Disulfide unruptured" group); and (3) the hydrolysis of the disulfide bond in C1-df and the release of the sequestered peptide sequences upon stretching (denoted as the "Disulfide ruptured" group). The representative force-extension curves of the three groups are shown in Fig. 5c-e (Supplementary Note 4). Unlike the case of ester bond, which forms in all $\mathrm{C} 1$ domains, the disulfide bond is only formed in $86.7 \%$ of the total events (168 out of 193) (Fig. 5f). Among them, the "Disulfide ruptured" events only account for $4.3 \%$ (16 out of 389 ) of the total events (Fig. 5f). However, in the absence of reducing agent, this group is missing. This indicates that the disulfide bond in C1$\mathrm{df}$ is also protected from the attack of the reducing agent (Supplementary Fig. 17). To further confirm this, we studied the mechanical unfolding of the circular permutant of $\mathrm{C} 1$ - df $\left(\mathrm{Cl}_{\mathrm{CP}^{-}}\right.$ df), by replacing the ester bond in $\mathrm{Cl}_{C P}$ to disulfide bond (Fig. 6). As in $\mathrm{Cl}_{C P}$-df, the partially unfolded protein structure cannot prevent the attack from TCEP, the rupture of disulfide bonds was observed in $42.7 \%$ of the total events. Even without TECP, in $3.1 \%$ of the traces, we observed the rupture of disulfide bonds at forces of $\sim 2 \mathrm{nN}$. Taken together, our results indicated that the $\mathrm{C} 1$ structure can efficiently prevent the reducing of the imbedded disulfide bond in the reducing environment under forces, similar to the protecting effect for the ester bond in $\mathrm{C}_{W T}$. This contrasts with other disulfide bond containing proteins studied previously $49,51,52$. In those proteins, the disulfide bond was caged in folded protein structures and can be decaged by mechanical unfolding the proteins. Note that other reducing agents, such as L-glutathione hydrochloride, $\beta$-mercaptoethanol, and 1,4-DLdithiothreitol, can also reduce the disulfide bonds. As they are of different size and reduce disulfide bonds following different mechanisms ${ }^{53}$, the shielding effect of the $\mathrm{C} 1$ structure for these reducing agents may be different from that for TCEP.

\section{Discussion}

By employing single-molecule force spectroscopy, protein engineering, and molecular dynamics simulation, we studied the nanomechanical properties of the $\mathrm{C} 1$ domain of $\mathrm{Cpe} 0147$ from Clostridium perfringens with a spontaneously formed intramolecular ester bond. Our results revealed that $\mathrm{C} 1$ can retain a partially unfolded structure under a force as high as $2.5 \mathrm{nN}$. When the ester bond was removed, the mechanical stability of the protein $\mathrm{C1}_{T 11 A}$ dropped sharply with unfolding forces of $\sim 92 \mathrm{pN}$. This demonstrates the important role of covalent ester bond for the mechanical stability of $\mathrm{C} 1$. However, using a circular permutation of $\mathrm{C} 1\left(\mathrm{Cl}_{C P}\right)$, we revealed that the protein can first partially unfold at a force of $\sim 90 \mathrm{pN}$ and then rupture the ester bond at a force of $\sim 80 \mathrm{pN}$. Although all intramolecular interactions are kept in $\mathrm{Cl}_{C P}$, the change of the force orientation can dramatically affect the mechanical response. These results highlight the importance of the interplay of the protein structure and the ester bond to the ultrahigh mechanical stability: The ester bond locates at the force concentration point to lock the protein structure to a partially unfolded state with most of the native contacts preserved. On the other hand, the protein structure prevents the ester bond from water attack and makes this chemically labile bond mechanically stable. This stabilization mechanism was verified using molecular dynamics simulation. Moreover, by using disulfide bond mutants, we showed that the unique correlation of the protein structure and the location of the intramolecular bond is responsible to the ultrahigh mechanical stability of the protein domain, regardless what type of bonds at the force concentration point. This can also explain that the ester bond containing proteins show similar mechanical properties as the isopeptide bond containing proteins with similar structures.

Previous bioinformatics analysis suggested that this type of ester bonds is conserved in many cell surface proteins of Grampositive bacteria. Moreover, these ester bond containing proteins share similar structures despite that they show very low sequence 
a

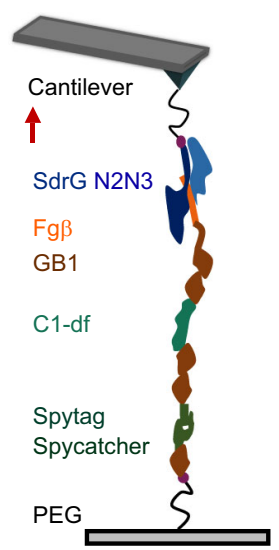

C

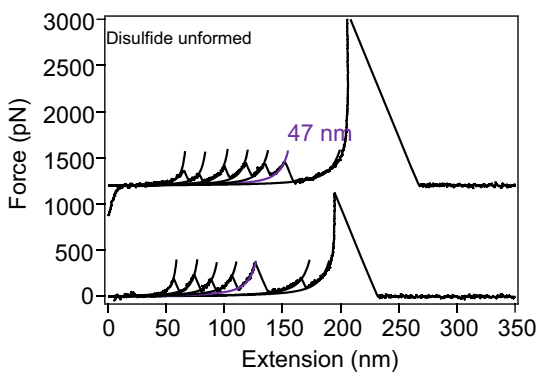

e

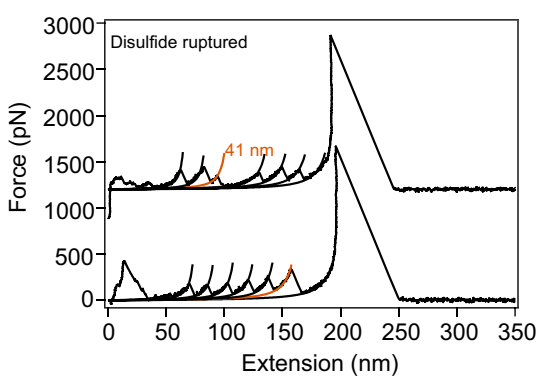

b
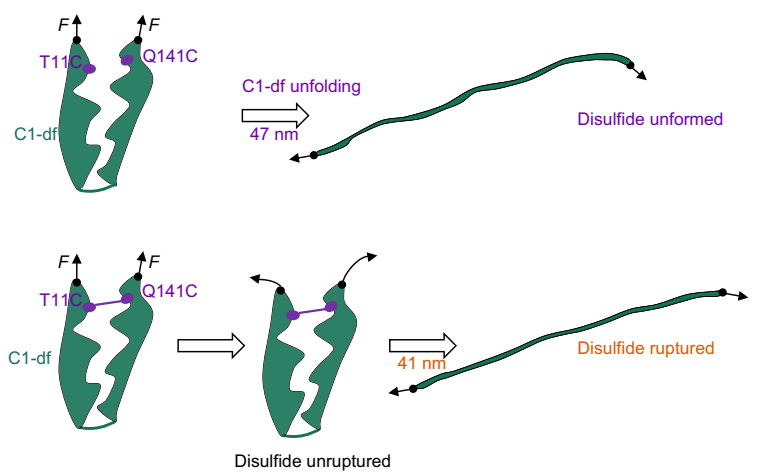

d

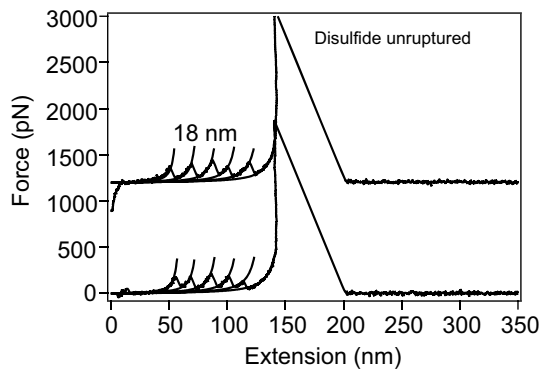

f

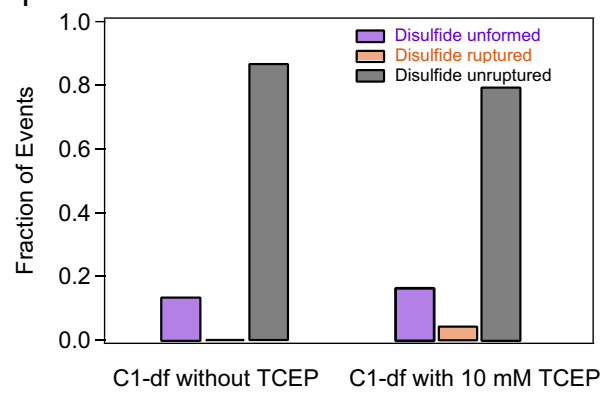

Fig. 5 Mechanical unfolding of C1-df. a Schematic of the AFM-based single-molecule force spectroscopy experiments. Polyprotein Fg $\beta$-(GB1) ${ }_{2}$-C1-df$(\mathrm{GB1})_{2}$-Spytag was linked to the cys-GB1-Spycatcher modified substrate covalently through the Spycatcher/Spytag chemistry and picked up by the SdrGcys modified cantilever through the Fg $\beta / \mathrm{SdrG}$ interaction. Thus, the force-extension curves should contain five GB1 fingerprints. b Three possible unfolding/hydrolysis pathways of C1-df under load and the corresponding contour length change. c Representative force-extension curves of the "Disulfide unformed" group. Two populations of unfolding events as showed, five peaks in black related to GB1 unfolding and the purple one with $\Delta L_{c}$ of $47 \mathrm{~nm}$ corresponds to the unfolding of C1-df. d Representative force-extension curves of the "Disulfide unruptured" group. The traces show only five GB1 unfolding events without the signature of C1-df unfolding or disulfide bond rupture. e Representative force-extension curves of the "Disulfide ruptured" group. Except for the five GB1 unfolding events, there is an additional peak with $\Delta L_{c}$ of $41 \mathrm{~nm}$ (colored in orange) corresponding to the rupture of the disulfide bond. $\mathbf{f} 2 \mathrm{D}$ bar shows the relative populations of the three kinds of events without (left, $n=193$ ) or with (right, $n=389$ ) 10 mM TCEP. This experiment was repeated 4 times independently with similar results. Source data are provided as a Source Data file.

homology ${ }^{16}$. We hypothesized that these proteins also exhibit high mechanical stabilities. To confirm this, we studied the mechanical unfolding of another ester bond containing protein ParV from the Gram-positive bacterium, Parvimonas sp. (Supplementary Fig. 19a, b). Similar to the C1 domain of Cpe0147, we did not observe any unfolding signatures of wide type ParV up to a stretching force of $2 \mathrm{nN}$ (Supplementary Fig. 19c). Once the ester bond was deleted by mutation, the protein unfolded at forces of $\sim 200 \mathrm{pN}$ (Supplementary Fig. 19d, e). Therefore, we propose that the chemically labile ester bonds play similar roles as isopeptide bonds to stabilize the structure of Gram-positive surface proteins. In contrast, the structures and the position of the intramolecular bonds of the surface proteins containing thioester are distinct from the proteins containing ester or isopeptide bonds. They are evolved for surface anchoring instead of stabilizing the protein structures ${ }^{12}$.

Why has Cpe0147 evolved such exceptional mechanical stabilities? As one of the major putative MSCRAMMs of C. perfringens, it anchors to the cell wall through its C-terminus and projects its $\mathrm{N}$-terminal adhesion domain through 11 repeat ester bond containing Ig domains. The internal ester bonds are strategically positioned to covalently link the first and last $\beta$-strands of these Ig domains ${ }^{9}$. Therefore, the stress on the proteins is only propagated from the first $\beta$-strand, through the ester bond, and to the final $\beta$-strand ( $\beta 7)$, leaving the rest of the protein bypassed by the mechanical forces. Mechanical unfolding of these domains may affect the self-assembly and function of these domains or even make them susceptible to proteolytic attack. Their high 
a

b

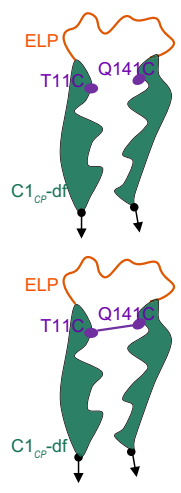

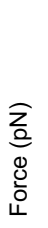
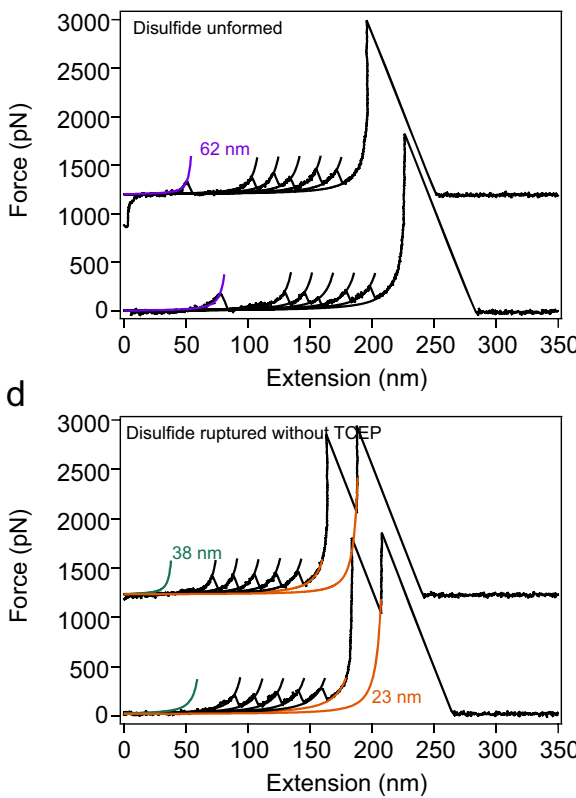

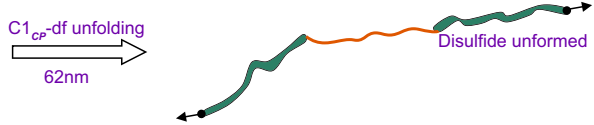

Partial $\mathrm{C} 1_{C P}$-df unfolding

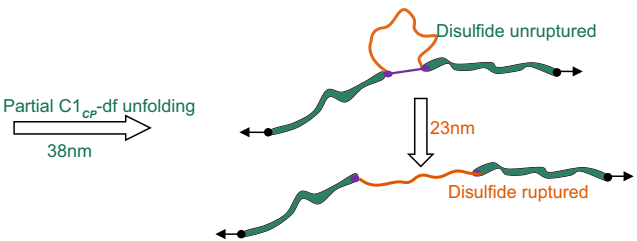

C

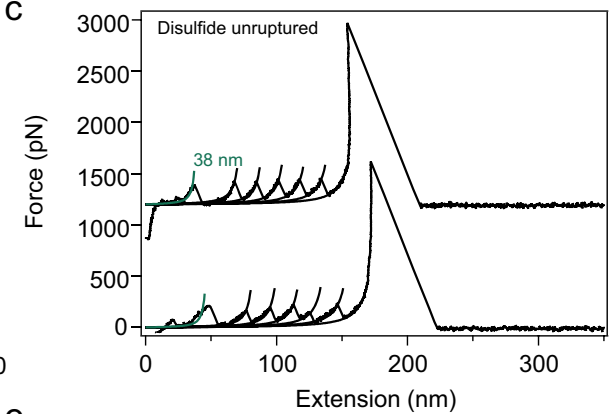

e

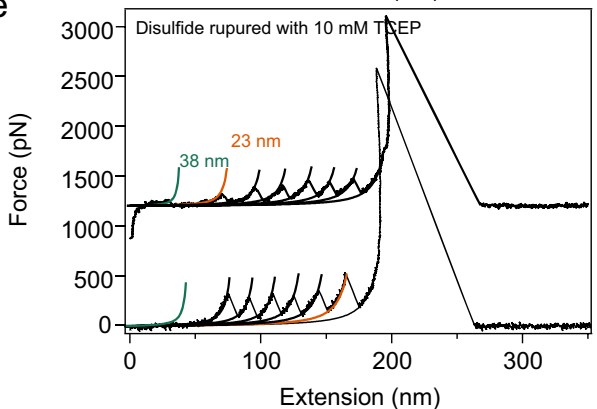

f

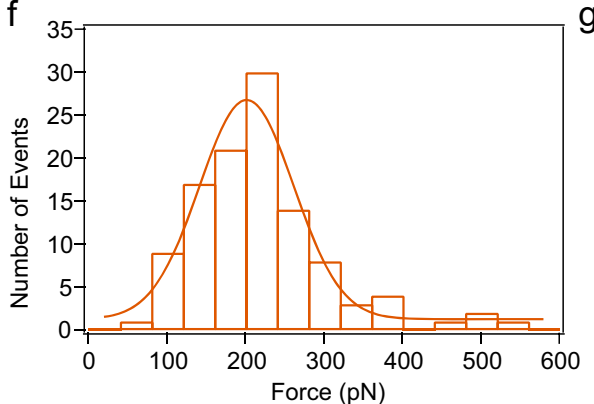

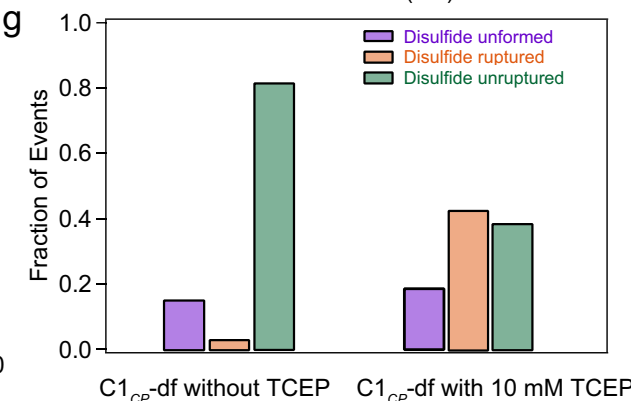

Fig. 6 Mechanical unfolding of $\mathrm{C1}_{C P}$-df. $\mathrm{C1}_{C P}$-df was constructed in the same way as $\mathrm{C1}_{C P}$ except that the ester bond was replaced by a disulfide bond. a Three possible unfolding/hydrolysis pathways of $\mathrm{Cl}_{C P}$-df under load and the corresponding contour length change. $\mathbf{b}$ Representative force-extension curves of the "Disulfide unformed" group. c Representative force-extension curves of the "Disulfide unruptured" group. Representative force-extension curves of the "Disulfide ruptured" group in PBS with (d) and without (e) $10 \mathrm{mM}$ TCEP. $\mathbf{f}$ Histogram of the mechanical rupture force of disulfide bond in PBS with $10 \mathrm{mM}$ TCEP. Fitting the force distribution with Monte Carlo simulation results in a $\Delta \mathrm{x}_{\mathrm{u}}$ of $0.13 \mathrm{~nm}$ and o of $0.39 \mathrm{~s}^{-1}$ (Supplementary Fig. 18). $\mathbf{g} 2 \mathrm{D}$ bars show the relative populations of the three kinds of events without (left, $n=844$ ) or with $10 \mathrm{mM}$ TCEP (right, $n=754$ ). This experiment was repeated more than five times independently with similar results. Source data are provided as a Source Data file.

mechanical stability allows them to maintain folded structures even under mechanical forces of more than $2 \mathrm{nN}$, which is critical to their biological functions.

Besides strong binding, bacteria also need to develop a mechanism to release the mechanical load to migrate and spread over tissue surfaces for colonization. Especially on wound tissues, the infected bacteria can proliferate quickly and cause inflammation. The wound sites typically show basic $\mathrm{pH}$ and low salt concentrations ${ }^{54}$. Previous biochemical studies indicate that the basic $\mathrm{pH}$ destabilizes the $\mathrm{C} 1$ domain and promotes the hydrolysis of ester bonds ${ }^{25}$. Moreover, the $\mathrm{C} 1$ contains two calcium binding sites $^{16}$. Although these calcium binding sites do not contribute to the thermodynamic stability or the ester bond formation ${ }^{23}$, they can change the protein local structural flexibilities. We therefore studied whether the mechanical stability of $\mathrm{Cl}_{W T}$ is affected by some unusual pathological conditions. We pulled the $\mathrm{Fg} \beta$ $(\mathrm{GB} 1)_{2}-\mathrm{Cl}_{W T^{-}}(\mathrm{GB} 1)_{2}$-cys following the same experimental scheme as that shown in Fig. 1 under a basic $\mathrm{pH}$ of 9.0 or in the presence of a calcium chelation ligand, ethylenediaminetetraacetic acid (EDTA) to remove the calcium ions. Majorities of the 
a

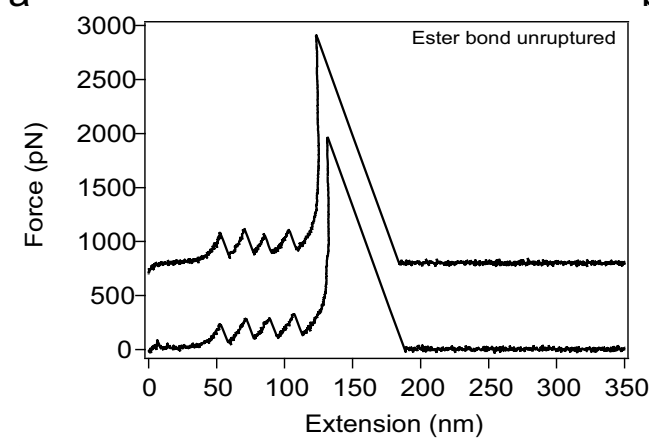

C

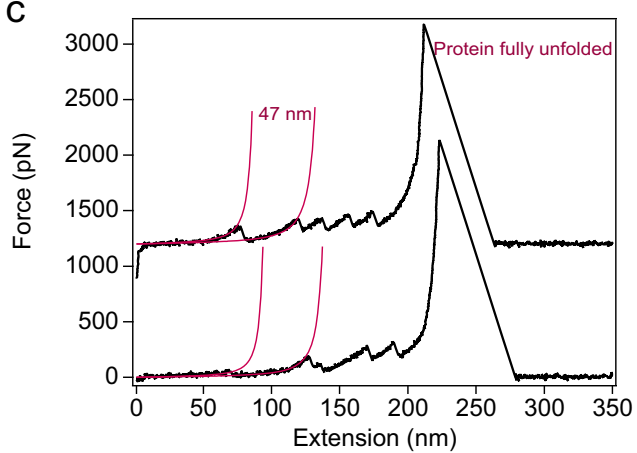

b

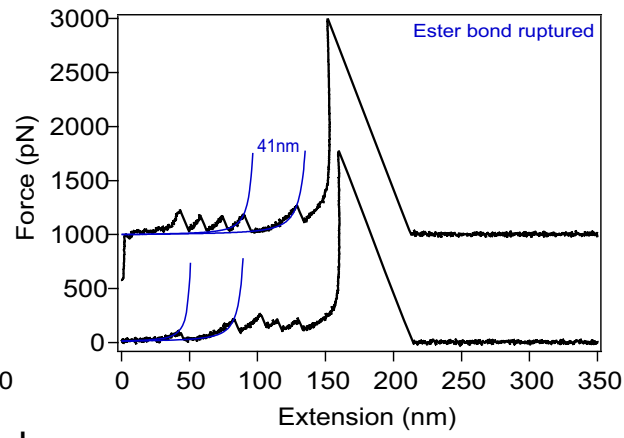

d

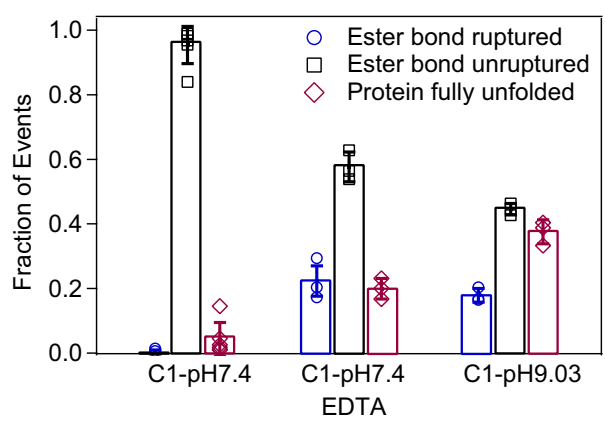

e
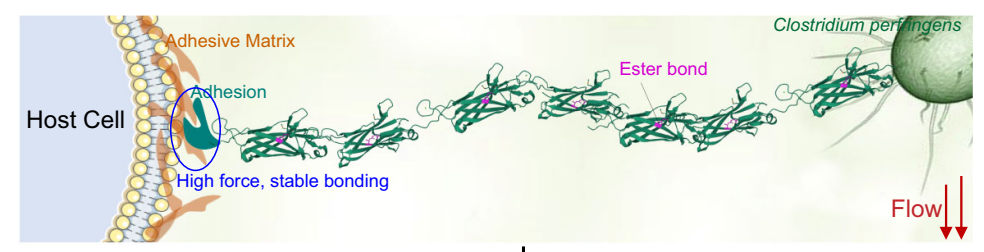

High pH/ Low concentration of calcium

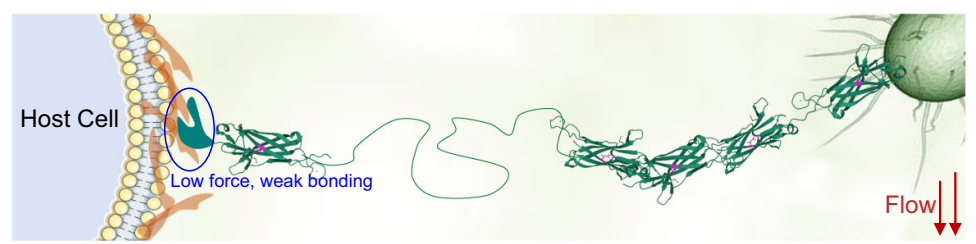

Fig. $\mathbf{7}$ Mechanical features of $\mathbf{C 1}$ at a basic pH of $\mathbf{9 . 0}$ and in the presence of EDTA (10 $\mathbf{~ m M ) . ~ a ~ R e p r e s e n t a t i v e ~ f o r c e - e x t e n s i o n ~ c u r v e s ~ o f ~ t h e ~ " E s t e r ~ b o n d ~}$ unruptured" group. The traces show only four GB1 unfolding events without the signature of C1 unfolding or ester bond rupture. $\mathbf{b}$ Representative force-extension curves of the "Ester bond ruptured" group. Two populations of unfolding events were observed: Four peaks with a $\Delta L_{c}$ of $41 \mathrm{~nm}$ relate to GB1 unfolding and an additional one with a $\Delta L_{c}$ of $41 \mathrm{~nm}$ corresponds to the rupture of the ester bond. c Representative force-extension curves of the "Protein fully unfolded" group. Except for the four GB1 unfolding events, there is an additional peak with $\Delta L_{c}$ of $47 \mathrm{~nm}$ (colored in red, upper trace) or a spacer corresponding to the unfolded of $\mathrm{C} 1$ (lower trace). $\mathbf{d}$ The statistics of the three types of events of $\mathrm{C} 1$ at $\mathrm{pH} 7.4$ and pH 9.0 and in the presence of EDTA ( $\mathrm{pH}$ 7.4). The numbers of tests in three conditions are 3,3,3 for $\mathrm{pH} 7.4, \mathrm{pH} 7.4$ with EDTA and pH 9, respectively. Error bars represent SD. e Proposed mechanism for the function of ester bond containing proteins during bacteria invasion. This experiment was repeated three times independently with similar results. Source data are provided as a Source Data file.

force-extension curves were still the same as that under the normal conditions without showing any $\mathrm{C} 1$ domain unfolding or ester bond hydrolysis features (Fig. 7a). However, we observed that $\sim 17.9 \%$ events at $\mathrm{pH} 9.0$ and $\sim 22.3 \%$ events in the presence of EDTA showed clear ester bond rupture events with a contour length increment of $\sim 41 \mathrm{~nm}$ (Supplementary Fig. 20a), indicating that the structure of $\mathrm{Cl}$ becomes flexible under these conditions (Fig. 7b). As such, they cannot fully protect the ester bond from water attack. Moreover, we observed $\sim 37.5 \%$ events at $\mathrm{pH} 9.0$ and $\sim 20.0 \%$ events in the presence of EDTA showed the unfolding of the full length $\mathrm{C} 1$ domain without the intramolecular ester bond (Fig. 7c). These events either showed a peak with a contour length increment of $\sim 47 \mathrm{~nm}$ (Fig. $7 \mathrm{c}$, upper trace, Supplementary Fig. 20b) or showed a long spacer before the first GB1 unfolding peak because the $\mathrm{C} 1$ domain was already unfolded before being stretched in the polyprotein (Fig. 7c, lower trace). For comparison, the probability of the ester bond rupture and the $\mathrm{C} 1$ domain unfolding events under $\mathrm{pH} 7.4, \mathrm{pH} 9.0$, and in the presence of EDTA are summarized in Fig. 7d and Supplementary Tables 1 and 3. Clearly, the pathological conditions can destabilize the $\mathrm{C} 1$ domain. It is worth mentioning that in the pathological conditions of slightly basic $\mathrm{pH}$ and low calcium concentrations, the $\mathrm{C} 1$ domain may still be fully folded, which is different from the experimental conditions in which the $\mathrm{pH}$ was set as 9.0 and the calcium ions were almost completely removed by the addition of EDTA.

We propose that destabilizing the stalk region of Cpe0147 may be beneficial for the colonization of the bacteria (Fig. 7e). The 
N-terminal tip adhesion of cpe 0147 contains a thioester bond which can reversibly bind to fibronectin through force regulation. In recent work by Alonso-Caballero et al., they showed that the reactivity of a structural analog of this domain for surface binding is modulated by force-dependent protein folding/unfolding 24 . It undergoes reversible binding and unbinding to surface ligands under forces $<6 \mathrm{pN}$ and remains covalent attachment under higher mechanical stress. Unlike the tip adhesin, the stability of the stalk domains is critical for the force propagation to the tip adhesin. When the Ig-fold of the stalk domains is folded, they can efficiently transduce forces to the tip adhesin for strong surface anchoring. When they are partially unfolded, they can release the force on tip adhesin and promote its detachment from the tissue surfaces for migration. Therefore, the type and the position of the intramolecular bonds in the tip adhesin and the stalk domains are distinct. However, they are both evolved for efficient host surface binding, biofilm formation, and immune evasion. Further experimental work is needed to validate this hypothesis.

It remains unknown whether Cpe0147 can bind to the host or other cell adhesion proteins via the ester bond containing stalk region $^{55}$. If so, we hypothesize that the force direction can be altered by such binding events and eventually cause the local unfolding of the protein structure and expose the ester bond to water, similar to the circular permutant of $\mathrm{C} 1$ studied in this work. This can also lead to the hydrolysis of the ester bond, then complete unfolding of these domains, and eventually loss of adhesion. In contrast, the isopeptide bond containing proteins can only partially unfold in this scenario and refold back after the release of force due to the high stability of isopeptide bonds.

In summary, we employed AFM-based single-molecule force microscopy, protein engineering, and molecular dynamics simulation to study the mechanical properties of an ester bond containing protein, the $\mathrm{C} 1$ domain of Cpe0147 from Grampositive bacterium, $C$. perfringens. We find that despite that individual ester bond is mechanically much weaker than an isopeptide bond, $\mathrm{C} 1$ exhibits similar mechanical stability as the isopeptide bond containing protein and does not completely unfold even under forces $>2 \mathrm{nN}$. The ester bond locks the structure to a partially unfolded conformation, in which the ester bond remains largely water inaccessible. This allows the ester bond to withstand considerable mechanical forces and in turn prevent complete protein unfolding. Breaking this structural correlation by circular permutation leads to sequential protein unfolding and ester bond hydrolysis. Destabilizing the protein structure at basic $\mathrm{pHs}$ or low calcium concentrations also reduces the protecting effect and leads to the hydrolysis of the ester bond. We propose that the environment-dependent mechanical response of Cpe0147 may allow the bacteria to smartly switch between binding and unbinding states for better invasion. We further highlight the importance of the position of ester bond on the high mechanical strength of the protein by engineering a mutant with a disulfide bond at the ester bond location that can retain high mechanical stability in the presence of reducing agents. These results provide the molecular mechanism on the high mechanical stability of ester bonding containing protein and might also inspire the design of new antibiotics by mechanically destabilizing these proteins.

\section{Methods}

Chemicals. All chemicals used were supplied by Sigma-Aldrich (USA) or New England Biolabs (USA) if not specified explicitly.

Protein engineering. The genes for $\mathrm{C} 1$ domain of Cpe0147 from C. perfringens, Fgß/SdrG N2N3 domain from Staphylococcus epidermidis, and ParV from Parvimonas sp. were custom synthesized codon-optimized for expression in Escherichia Coli (GenScript, China), and designed to include $5^{\prime}$ BamHI, 3' BglII, and KpnI restriction sites. The genes encoding the chimeras used in this work were constructed in the pQE80L vector (Qiagen, Germany) or pET22b vector (Novagen, USA) based on the same sticky end generated by BamHI and BglII following standard molecular biology techniques ${ }^{56}$. Point mutations and circular permutations were created through polymerase chain reactions. The constructed plasmids were sequenced to confirm the correct protein sequence. All these constructs and full amino acid sequences are shown in Supplementary Information.

Protein expression and purification. All proteins used in this work were expressed in E. coli (BL 21) cells and purified with $\mathrm{Co}^{2+}$ affinity chromatography using TALON resins (Takara, USA). Purified protein samples were dialyzed to remove the imidazole that used for protein elusion and stored in PBS buffer at $4{ }^{\circ} \mathrm{C}$ until use. Protein concentrations were measured by spectrophotometry at $280 \mathrm{~nm}$ with typical final concentrations of $\sim 100 \mu \mathrm{M}$ (Thermo NanoDrop 2000, USA).

AFM sample preparation. More detailed AFM-based single-molecule force spectroscopy protocol has been published previously ${ }^{28,39}$. In brief, AFM cantilevers (Bruker, MLCT, USA) and glass surfaces (Sail Brand, China) were modified with aminosilane.

Glass surface: Glass substrates were cut into $1 \times 1 \mathrm{~cm}^{2}$ slides, soaked in a freshly prepared chromic mixture overnight, thoroughly washed with deionized (DI) water, ethanol, and acetone successively, and then dried under a steam of nitrogen to produce surfaces with exposed hydroxyl groups. These substrates were immersed in an anhydrous toluene solution containing 1\% (v/v) APTES (Merck, USA) at room temperature (R.T.) for $1 \mathrm{~h}$ for amination. Then, they were washed with toluene and ethanol, dried under a nitrogen flow. Finally, surfaces were incubated at $90^{\circ} \mathrm{C}$ for $30 \mathrm{~min}$. Glass substrates were stored in a desiccator under argon and typically used within half a month.

Cantilevers: Silicon nitride $\left(\mathrm{Si}_{3} \mathrm{~N}_{4}\right)$ cantilevers (MLCT-D, Bruker) were first cleaned with Milli-Q water, and then placed in a chromic mixture (chromic acid) at $80^{\circ} \mathrm{C}$ for $30 \mathrm{~min}$. After that, the cantilevers were washed with DI water, then ethanol, and dried under a steam of nitrogen. Then, the hydroxylated cantilevers were immersed in an anhydrous toluene solution containing 1\% (v/v) APTES for $1 \mathrm{~h}$. After that, they were rinsed with toluene, then ethanol, dried under a nitrogen stream, and incubated at $80^{\circ} \mathrm{C}$ for $45 \mathrm{~min}$. Finally, they were stored overnight under argon and used in the following steps.

Both glass substrates and cantilevers were immersed in DMSO containing $0.2 \mathrm{mM}$ Mal-PEG-NHS (MW: $5000 \mathrm{Da}$, Nanocs, USA) for $1 \mathrm{~h}$. After being washed with DMSO, ethanol, and dried under a nitrogen stream, the resulting maleimidecoated glass substrates and cantilevers were kept dry at $-20^{\circ} \mathrm{C}$ before use in the following protein modification steps in the single-molecule experiments.

AFM-based single-molecule force spectroscopy. The force spectroscopy experiments were carried out using a commercial JPK ForceRobot 300 AFM system (JPK Instruments AG, Germany). Experiments were conducted at R.T. $\left(22^{\circ} \mathrm{C}\right)$ and performed in $10 \mathrm{mM}$ PBS buffer with or without $10 \mathrm{mM}$ TCEP if needed. Soft silicon nitride MLCT-D cantilevers of typical spring constant of $30-45 \mathrm{pN} \mathrm{nm}^{-1}$ were used for all experiments and calibrated using the thermal tune method using the data acquisition software from JPK after allowing the cantilever to equilibrate in solution for at least $30 \mathrm{~min}$. To transform the electrical signals from the photodiode to the actual displacement, the optical lever sensitivity and its inverse was determined by pushing the cantilever tip to the glass substrate and assumed the bending of the cantilever equal to the movement of the piezoelectric positioner. We did not correct the bending angles of the cantilever, which may lead to $\sim 10 \%$ errors to the estimated spring constants $\mathrm{s}^{57}$. In a typical pulling experiment, cantilevers were briefly and gently $(\sim 300 \mathrm{pN})$ brought in contact with the functionalized surface and held at the surface for $0.5 \mathrm{~s}$, then retracted at a constant velocity of $1.6 \mu \mathrm{m} \mathrm{s}^{-1}$. The sampling frequency was $10 \mathrm{kHz}$ and the records were smoothed by the moving average of 10 points. The force-extension curves were recorded using JPK data processing software and were further analyzed by a custom-written procedure in Igor 6.37 (Wavemetric Inc).

We only used the curves containing four peaks of contour length increments of $\sim 18 \mathrm{~nm}$ corresponding to the unfolding of the GB1 domains for data analysis. The contour length increment was determined by fitting the force peaks using the worm-like chain model with persistence lengths in a range of $\sim 0.2-0.5 \mathrm{~nm}$. The noise level of the force-extension curves was $\sim 10 \mathrm{pN}$. Only the peaks with rupture forces higher than three times the noise level were included in the force histogram. The contour length increment was determined by fitting the consecutive peaks using the WLC model with fitting residuals $<20 \mathrm{pN}$. In the cases that the traces only show a spacer instead of a detectable peak, we assigned them as the signature of the unfolded protein domain, because the unfolding of the protein structure is the prerequisite for the hydrolysis of the ester bond.

Molecular dynamics simulations. The molecular dynamics simulations were conducted by the GROMACS 2019 software ${ }^{58}$ with the ff 14 SB force field ${ }^{59}$ and TIP3P water ${ }^{60}$. To model the ester bond, we introduced a covalent bond between the Thr-11 (O $\gamma 1)$ and Gln-141 (C $)$. The extra atoms (H $\gamma 1$ of Thr-11; Ne2, He21, and $\mathrm{H} \varepsilon 22$ of Gln-141) were removed, with their partial charges being integrated into the nearby heavy atoms. The atomic coordinates of the $\mathrm{Cl}_{W T}$ were taken from the Protein Data Bank (entry $4 \mathrm{MKM})^{16}$. The five N-terminal residues lacking 
structural information were not included. In constructing the $\mathrm{Cl}_{C P}$, a ELP linker with the length of 20 amino acids was added between the two termini of the $\mathrm{Cl}$, and the residues Leu-125 and Asp-126 were used as the new termini. The threedimensional structure of the linker was modeled by the ModLoop ${ }^{61,62}$.

The $\mathrm{Cl}_{W T}$ and $\mathrm{Cl}_{C P}$ were solvated in the rectangular water boxes with the dimensions of $\sim 241 \AA \times 84 \AA \times 85 \AA$ (with 48891 water molecules) and $1017 \AA \times 74 \AA \times 74 \AA$ (with 159145 water molecules), respectively. Sodium ions were added to neutralize the systems. The LINCS algorithm was used to restrain the covalent bond involving hydrogen atoms ${ }^{63}$. After a 50,000-step minimization using the steepest descent method, each system was equilibrated for $0.1 \mathrm{~ns}$ in the NVT ensemble and another $0.1 \mathrm{~ns}$ in the NPT ensemble. The temperature and pressure were controlled at $298.0 \mathrm{~K}$ and $1.0 \mathrm{~atm}$, respectively. The heavy atoms of the proteins were restrained to their original positions by a harmonic potential during the minimization and equilibrium steps. Starting from the equilibrated structures, we performed steered MD simulations by applying pulling force between the termini residues along the $\mathrm{x}$-axis in NVT ensemble. For the $\mathrm{Cl}_{W T}$, the constant pulling force were applied to the termini residues with the strength of 0 and $1500 \mathrm{pN}$ and the simulations lasted for $50 \mathrm{~ns}$. For the $\mathrm{C} 1_{C P}$, we firstly conducted a constant velocity pulling simulations with the pulling speed of $49 \AA$ / ns, such that the ester bond stars to sustain pulling force. Then, we performed the same constant pulling force simulations as that in the $\mathrm{Cl}_{W T}$. Eight independent simulations were conducted for each system. To increase the statistics, we also performed another two (one) constant pulling force simulations with the length of $35 \mathrm{~ns}$ for the $\mathrm{Cl}_{W T}\left(\mathrm{Cl}_{C P}\right)$. The snapshots sampled during the constant pulling force simulations were used for analysis with the snapshots corresponding to the first $5 \mathrm{~ns}$ being omitted. The softwares MDTraj ${ }^{64}$ and PyMOL were used for the structure visualization.

Reporting summary. Further information on research design is available in the Nature Research Reporting Summary linked to this article.

\section{Data availability}

The source data underlying all figures are provided as Source Data file. All other data that support the findings of this study are available for download from https://biomech.nju. edu.cn/Download/index.html. Source Data are provided with this paper.

\section{Code availability}

The AFM code that supports the findings of this study is available from https://biomech. nju.edu.cn/Download/index.html.

Received: 25 November 2020; Accepted: 10 August 2021; Published online: 23 August 2021

\section{References}

1. Foster, T. J., Geoghegan, J. A., Ganesh, V. K. \& Hoeoek, M. Adhesion, invasion and evasion: the many functions of the surface proteins of Staphylococcus aureus. Nat. Rev. Microbiol. 12, 49-62 (2014).

2. Arciola, C. R., Campoccia, D. \& Montanaro, L. Implant infections: adhesion, biofilm formation and immune evasion. Nat. Rev. Microbiol. 16, 397-409 (2018).

3. Liu, Z. et al. High force catch bond mechanism of bacterial adhesion in the human gut. Nat. Commun. 11, 4321 (2020)

4. Mathelie-Guinlet, M. et al. Force-clamp spectroscopy identifies a catch bond mechanism in a Gram-positive pathogen. Nat. Commun. 11, 5431-5431 (2020).

5. Dufrene, Y. F. \& Persat, A. Mechanomicrobiology: how bacteria sense and respond to forces. Nat. Rev. Microbiol. 18, 227-240 (2020).

6. El-Kirat-Chatel, S., Beaussart, A., Mathelie-Guinlet, M. \& Dufrene, Y. F. The importance of force in microbial cell adhesion. Curr. Opin. Colloid . 47, 111-117 (2020).

7. Patti, J. M., Allen, B. L., McGavin, M. J. \& Hook, M. Mscramm-mediated adherence of microorganisms to host tissues. Annu. Rev. Microbiol. 48, 585-617 (1994).

8. Telford, J. L., Barocchi, M. A., Margarit, I., Rappuoli, R. \& Grandi, G. Pili in Gram-positive pathogens. Nat. Rev. Microbiol. 4, 509-519 (2006).

9. Schwarz-Linek, U. \& Banfield, M. J. Yet more intramolecular cross-links in Gram-positive surface proteins. Proc. Nati. Acad. Sci. USA 111, 1229-1230 (2014).

10. Baker, E. N., Squire, C. J. \& Young, P. G. Self-generated covalent cross-links in the cell-surface adhesins of Gram-positive bacteria. Biochem. Soc. Trans. 43, 787-794 (2015).

11. Dodds, A. W., Ren, X. D., Willis, A. C. \& Law, S. K. A. The reaction mechanism of the internal thioester in the human complement component C4. Nature 379, 177-179 (1996).
12. Echelman, D. J., Lee, A. Q. \& Fernandez, J. M. Mechanical forces regulate the reactivity of a thioester bond in a bacterial adhesin. J. Biol. Chem. 292, 8988-8997 (2017)

13. Walden, M. et al. An internal thioester in a pathogen surface protein mediates covalent host binding. Elife 4, e06638 (2015).

14. Kang, H. J., Coulibaly, F., Clow, F., Proft, T. \& Baker, E. N. Stabilizing isopeptide bonds revealed in Gram-positive bacterial pilus structure. Science 318, 1625-1628 (2007).

15. Alegre-Cebollada, J., Badilla, C. L. \& Fernandez, J. M. Isopeptide bonds block the mechanical extension of pili in pathogenic Streptococcus pyogenes. J. Biol. Chem. 285, 11235-11242 (2010).

16. Kwon, H., Squire, C. J., Young, P. G. \& Baker, E. N. Autocatalytically generated Thr-Gln ester bond cross-links stabilize the repetitive Ig-domain shaft of a bacterial cell surface adhesin. Proc. Nati. Acad. Sci. USA 111, 1367-1372 (2014).

17. Kang, H. J. \& Baker, E. N. Intramolecular isopeptide bonds give thermodynamic and proteolytic stability to the major pilin protein of Streptococcus pyogenes. J. Biol. Chem. 284, 20729-20737 (2009).

18. Hagan, R. M. et al. NMR spectroscopic and theoretical analysis of a spontaneously formed Lys-Asp isopeptide bond. Angew. Chem. Int. Ed. 49, 8421-8425 (2010)

19. Kang, H. J., Paterson, N. G., Gaspar, A. H., Ton-That, H. \& Baker, E. N. The Corynebacterium diphtheriae shaft pilin SpaA is built of tandem Ig-like modules with stabilizing isopeptide and disulfide bonds. Proc. Nati. Acad. Sci. USA 106, 16967-16971 (2009).

20. Keeble, A. H. \& Howarth, M. Power to the protein: enhancing and combining activities using the Spy toolbox. Chem. Sci. 11, 7281-7291 (2020).

21. Rivas-Pardo, J. A., Badilla, C. L., Tapia-Rojo, R., Alonso-Caballero, A. \& Fernandez, J. M. Molecular strategy for blocking isopeptide bond formation in nascent pilin proteins. Proc. Nati. Acad. Sci. USA 115, 9222-9227 (2018).

22. Echelman, D. J. et al. CnaA domains in bacterial pili are efficient dissipaters of large mechanical shocks. Proc. Nati. Acad. Sci. USA 113, 2490-2495 (2016).

23. Wang, B., Xiao, S., Edwards, S. A. \& Graeter, F. Isopeptide bonds mechanically stabilize Spy0128 inbacterial pili. Biophys. J. 104, 2051-2057 (2013)

24. Alonso-Caballero, A. et al. Protein folding modulates the chemical reactivity of a Gram-positive adhesin. Nat. Chem. 13, 172-181 (2021).

25. Young, P. G. et al. Harnessing ester bond chemistry for protein ligation. Chem Commun. 53, 1502-1505 (2017)

26. Akbulatov, S., Tian, Y., Kapustin, E. \& Boulatov, R. Model studies of the kinetics of ester hydrolysis under stretching force. Angew. Chem. Int. Ed. 52, 6992-6995 (2013)

27. Yu, H., Siewny, M. G. W., Edwards, D. T., Sanders, A. W. \& Perkins, T. T. Hidden dynamics in the unfolding of individual bacteriorhodopsin proteins. Science 355, 945-949 (2017).

28. Milles, L. F., Schulten, K., Gaub, H. E. \& Bernardi, R. C. Molecular mechanism of extreme mechanostability in a pathogen adhesin. Science 359, 1527-1532 (2018).

29. Mueller, D. J. \& Dufrene, Y. F. Atomic force microscopy as a multifunctional molecular toolbox in nanobiotechnology. Nat. Nanotechnol. 3, 261-269 (2008).

30. Lei, H. et al. Reversible unfolding and folding of the metalloprotein ferredoxin revealed by single-molecule atomic force microscopy. J. Am. Chem. Soc. 139, 1538-1544 (2017)

31. Milles, L. F. \& Gaub, H. E. Extreme mechanical stability in protein complexes. Curr. Opin. Struct. Biol. 60, 124-130 (2020).

32. Bao, Y., Luo, Z. \& Cui, S. Environment-dependent single-chain mechanics of synthetic polymers and biomacromolecules by atomic force microscopy-based single-molecule force spectroscopy and the implications for advanced polymer materials. Chem. Soc. Rev. 49, 2799-2827 (2020).

33. Milles, L. F., Unterauer, E. M., Nicolaus, T. \& Gaub, H. E. Calcium stabilizes the strongest protein fold. Nat. Commun. 9, 4764 (2018).

34. Cao, Y. \& Li, H. Polyprotein of GB1 is an ideal artificial elastomeric protein Nat. Mater. 6, 109-114 (2007).

35. Lei, H. et al. Stretchable hydrogels with low hysteresis and anti-fatigue fracture based on polyprotein cross-linkers. Nat. Commun. 11, 4032 (2020).

36. Cao, Y., Lam, C., Wang, M. J. \& Li, H. B. Nonmechanical protein can have significant mechanical stability. Angew. Chem. Int. Ed. 45, 642-645 (2006)

37. Zakeri, B. et al. Peptide tag forming a rapid covalent bond to a protein, through engineering a bacterial adhesin. Proc. Nati. Acad. Sci. USA 109, E690-E697 (2012)

38. Keeble, A. H. et al. Approaching infinite affinity through engineering of peptide-protein interaction. Proc. Nati. Acad. Sci. USA 116, 26523-26533 (2019).

39. Huang, W. et al. Maleimide-thiol adducts stabilized through stretching. Nat. Chem. 11, 310-319 (2019).

40. Grandbois, M., Beyer, M., Rief, M., Clausen-Schaumann, H. \& Gaub, H. E. How strong is a covalent bond? Science 283, 1727-1730 (1999).

41. Xue, Y., Li, X., Li, H. \& Zhang, W. Quantifying thiol-gold interactions towards the efficient strength control. Nat. Commun. 5, 4348 (2014). 
42. Lin, Y., Kouznetsova, T. B. \& Craig, S. L. A latent mechanoacid for timestamped mechanochromism and chemical signaling in polymeric materials. $J$. Am. Chem. Soc. 142, 99-103 (2020).

43. Tian, Y. et al. A polymer with mechanochemically active hidden length. J. Am. Chem. Soc. 142, 18687-18697 (2020).

44. Li, Y. et al. Molecular design principles of Lysine-DOPA wet adhesion. Nat. Commun. 11, 3895 (2020).

45. Delparastan, P., Malollari, K. G., Lee, H. \& Messersmith, P. B. Direct evidence for the polymeric nature of polydopamine. Angew. Chem. Int. Ed. 58, 1077-1082 (2019).

46. Song, G. et al. Single-molecule force spectroscopy reveals that the Fe-N bond enables multiple rupture pathways of the $2 \mathrm{Fe} 2 \mathrm{~S}$ cluster in a MitoNEET monomer. Anal. Chem. 92, 14783-14789 (2020).

47. Bernardi, R. C. et al. Mechanisms of nanonewton mechanostability in protein complex revealed by molecular dynamics simulations and singlemolecule force spectroscopy. J. Am. Chem. Soc. 141, 14752-14763 (2019).

48. Beyer, M. K. \& Clausen-Schaumann, H. Mechanochemistry: the mechanical activation of covalent bonds. Chem. Rev. 105, 2921-2948 (2005).

49. Ainavarapu, R. K. et al. Contour length and refolding rate of a small protein controlled by engineered disulfide bonds. Biophys. J. 92, 225-233 (2007).

50. Kosuri, P. et al. Protein folding drives disulfide formation. Cell 151, 794-806 (2012).

51. Wiita, A. P., Ainavarapu, R. K., Huang, H. H. \& Fernandez, J. M. Forcedependent chemical kinetics of disulfide bond reduction observed with singlemolecule techniques. Proc. Nati. Acad. Sci. USA 103, $7222-7227$ (2006).

52. Alegre-Cebollada, J., Kosuri, P., Andres Rivas-Pardo, J. \& Fernandez, J. M. Direct observation of disulfide isomerization in a single protein. Nat. Chem. 3, 882-887 (2011).

53. Ainavarapu, S. R. K., Wiita, A. P., Dougan, L., Uggerud, E. \& Fernandez, J. M. Single-molecule force spectroscopy measurements of bond elongation during a bimolecular reaction. J. Am. Chem. Soc. 130, 6479-6487 (2008).

54. Scalise, A. et al. Microenvironment and microbiology of skin wounds: the role of bacterial biofilms and related factors. Semin. Vasc. Surg. 28, 151-159 (2015).

55. Wren, B. W. A family of Clostridial and Streptococcal ligand-binding proteins with conserved C-terminal repeat sequences. Mol. Microbiol. 5, 797-803 (1991).

56. Lv, C. et al. Single-molecule force spectroscopy reveals force-enhanced binding of calcium ions by gelsolin. Nat. Commun. 5, 4623 (2014).

57. Rodriguez-Ramos, J. \& Rico, F. Determination of calibration parameters of cantilevers of arbitrary shape by finite element analysis. Rev. Sci. Instrum. 92, 045001 (2021)

58. Abraham, M. J. et al. GROMACS: high performance molecular simulations through multi-level parallelism from laptops to supercomputers. SoftwareX 1-2, 19-25 (2015).

59. Maier, J. A. et al. ff14SB: improving the accuracy of protein side chain and backbone parameters from ff99SB. J. Chem. Theory Comput. 11, 3696-3713 (2015).

60. Jorgensen, W. L., Chandrasekhar, J., Madura, J. D., Impey, R. W. \& Klein, M. L. Comparison of simple potential functions for simulating liquid water. $J$. Chem. Phys. 79, 926-935 (1983).

61. Fiser, A., Do, R. K. G. \& Sali, A. Modeling of loops in protein structures. Prot. Sci. 9, 1753-1773 (2000)

62. Fiser, A. \& Sali, A. ModLoop: automated modeling of loops in protein structures. Bioinformatics 19, 2500-2501 (2003).

63. Hess, B., Bekker, H., Berendsen, H. J. C. \& Fraaije, J. LINCS: a linear constraint solver for molecular simulations. J. Comput. Chem. 18, 1463-1472 (1997).
64. McGibbon, R. T. et al. MDTraj: a modern open library for the analysis of molecular dynamics trajectories. Biophys. J. 109, 1528-1532 (2015).

65. Gao, X. et al. Single-molecule experiments reveal the flexibility of a PerARNT-Sim domain and the kinetic partitioning in the unfolding pathway under force. Biophys. J. 102, 2149-2157 (2012).

\section{Acknowledgements}

This research is supported mainly by the National Key R\&D Program of China (grant no. 2020YFA0908100), National Natural Science Foundation of China (grants nos. $11804147,11974174,11934008,11974173)$, Youth Program of Natural Science Foundation of Jiangsu Province (grant no. BK20180335), and Fundamental Research Funds for the Central Universities (grant nos. 020414380154, 020414380080, 020414380148) and the project was supported by State Key Laboratory of Precision Measurement Technology and Instruments (Tianjin University) and the HPC center of Nanjing University.

\section{Author contributions}

Y.C., H.L. and W.W. conceived the project and designed the experiments. H.L. and Q.M. performed all the experiments and analyzed the data. W.L. designed and performed the simulations. J.W. and H.M. performed the quantum mechanics calculation of ester bond hydrolysis. Y.C., W.W. and M.Q. supervised the project. H.L. and Y.C. wrote the paper with contributions from all authors.

\section{Competing interests}

The authors declare no competing interests.

\section{Additional information}

Supplementary information The online version contains supplementary material available at https://doi.org/10.1038/s41467-021-25425-6.

Correspondence and requests for materials should be addressed to Y.C.

Peer review information Nature Communications thanks Yves Dufrene, Frauke Gräter and other, anonymous, reviewers for their contributions to the peer review of this work.

Reprints and permission information is available at http://www.nature.com/reprints

Publisher's note Springer Nature remains neutral with regard to jurisdictional claims in published maps and institutional affiliations.

Open Access This article is licensed under a Creative Commons Attribution 4.0 International License, which permits use, sharing, adaptation, distribution and reproduction in any medium or format, as long as you give appropriate credit to the original author(s) and the source, provide a link to the Creative Commons license, and indicate if changes were made. The images or other third party material in this article are included in the article's Creative Commons license, unless indicated otherwise in a credit line to the material. If material is not included in the article's Creative Commons license and your intended use is not permitted by statutory regulation or exceeds the permitted use, you will need to obtain permission directly from the copyright holder. To view a copy of this license, visit http://creativecommons.org/ licenses/by/4.0/.

(C) The Author(s) 2021 